\title{
Optical characterization of diode side pumped active elements*
}

\author{
JAN K. JABCZYṄSKI, JACEK KWIATKOWSKI, WALDEMAR ŻENDZIAN
}

Institute of Optoelectronics, Military University of Technology, ul. Sylwestra Kaliskiego 2, 00-908 Warszawa, Poland, e-mail: jjabczynski@wat.waw.pl.

\begin{abstract}
A numerical model of side pumped gain elements of rod or slab shape enabling estimation of overlapping efficiency and threshold was developed. This model was applied for analysis and optimization of laser heads side pumped by a single diode bar. The diode side pumped lasers heads for Nd:YAG rod and slab active elements were designed and characterized under real pumping conditions with a $40-\mathrm{W}$ diode bar. The optical performance of both types of heads was shown experimentally to be delimited by transversal, asymmetrical thermal gradients resulting in high level of depolarization and diffraction losses. The fundamental mode limit is about $20 \mathrm{~W}$ per $1 \mathrm{~cm}$ for a head side pumped by a single diode bar.
\end{abstract}

Keywords: diode pumping, slab lasers.

\section{Introduction}

Developed in the last decade the diode pumped lasers with output powers in the range from 10 to $20 \mathrm{~W}$ can be found in the wide areas of commercial and scientific applications. According to results of elementary theory of diode pumped lasers (see, e.g., [1], [2]) an end pumping scheme is applied in such type of lasers. The end pumping scheme (including pumping by two ends and periodic resonator concepts) enables achieving several dozen watts in circular fundamental mode beam with optical efficiency approaching $40-50 \%$. The main limitation of such scheme is the critical heat source density deposited in the gain medium by absorption of focused pump beam [3]. This effect leads to thermally induced deformation, stresses in the gain medium up to mechanical fracture. Moreover, the diodes destined for end pumping require application of an expensive, sophisticated beam shaping technique [4]. Thus, an application of side pumping scheme for such a range of output powers constitutes a reasonable alternative [5]-[10].

\footnotetext{
*This work was presented at XIII Polish-Czech-Slovak Optical Conference in Krzyżowa, September
} 2002. 


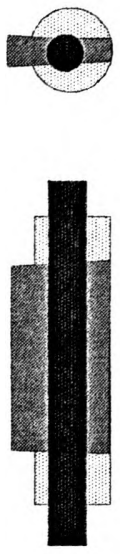

a
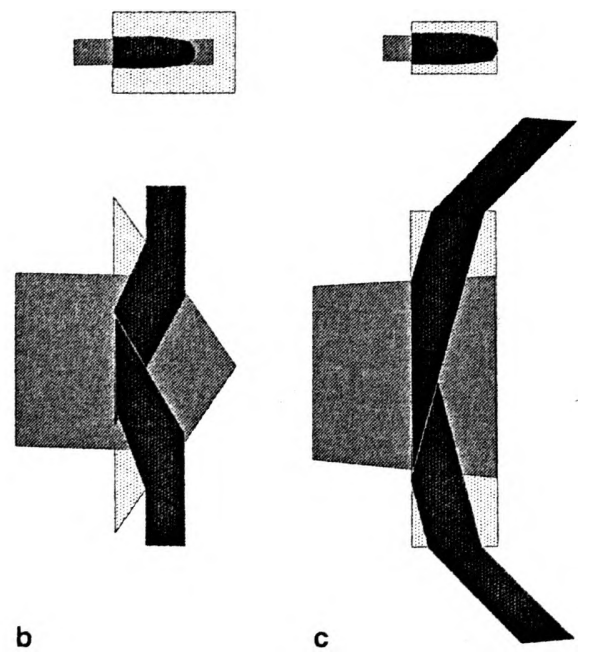

Fig. 1. Types of side pumped schemes under investigation: a-cylindrical rod (diameter/length $3 \times 15 \mathrm{~mm}$ ), b - Brewster cut triangle slab $\left(5 \times 5 \times 18 \mathrm{~mm}^{3}\right)$, c - "brick" rectangular rod $\left(2.5 \times 3 \times 20 \mathrm{~mm}^{3}\right)$.

The paper is aimed at theoretical analysis of the optical performance of three different side pumped schemes (see Fig. 1) and experimental verification of side pumped laser heads developed at the Institute of Optoelectronics, Military University of Technology, Warszawa, Poland. Numerical estimations and experiments were carried out using Nd:YAG crystal as an active medium. In Section 2, the results of theoretical analysis are presented. The investigations of temperature profiles, depolarization effects and energetic characteristics are given in the next three sections. In the last part, some conclusions are drawn.

\section{Analysis of pumping geometry}

According to elementary theory of diode pumped lasers [1], [2] the output power $P_{\text {out }}$ and threshold $\boldsymbol{P}_{\text {thr }}$ are given by:

$$
\begin{aligned}
& P_{\text {out }} \propto \eta_{\text {ovl }} \eta_{\mathrm{abs}}\left(P_{\mathrm{p}}-P_{\mathrm{thr}}\right), \\
& P_{\mathrm{thr}} \propto \frac{I_{\mathrm{sat}}}{\eta_{\mathrm{abs}}} V_{\mathrm{efc}}
\end{aligned}
$$

where $P_{\mathrm{p}}$ is the pump power, $\eta_{\mathrm{abs}}$ is the absorption efficiency given by

$$
\eta_{\mathrm{abs}}=1-\exp \left(-\alpha_{\mathrm{avg}} L\right)
$$

( $\alpha_{\text {avg }}$ is the averaged absorption coefficient of pump beam, $L$ is the length of active medium), while $\eta_{\text {ovl }}$ is the mode matching efficiency expressed by: 


$$
\eta_{\mathrm{ovl}}=\frac{\left(\int S_{l} G_{p} \mathrm{~d} V\right)^{2}}{\int S_{l}^{2} G_{p} \mathrm{~d} V},
$$

$V_{\text {efc }}$ is the effective mode volume

$$
V_{\text {efc }}=\frac{1}{\int S_{l} G_{p} \mathrm{~d} V}
$$

$I_{\text {sat }}=h v / \sigma \tau$ is the saturation power density ( $h$ - the Planck's constant, $v-$ the laser frequency, $\sigma$ - the emission cross-section, $\tau$ - the laser upper level lifetime), $S_{l}$ is the normalized 3D laser mode intensity distribution and $G_{p}$ is the normalized 3D gain distribution. We assume that gain distribution is proportional to absorbed pump density and laser mode intensity distribution corresponds to symmetrical Gaussian beam with the given waist radius $W_{0}$. Note that the following effects have been neglected: gain and thermally induced changes of fundamental mode, pump dependent cavity losses, back reflection of a pump beam on the rear surface of an element. For simplicity of analysis (given satisfactory explanation for Rayleigh range of cavity much longer than the pump volume size), we assume that inside the pump volume the mode distribution does not change along optical axis. Thus, we have for a given geometry of active elements the following parameters: mode radius $W_{0}$, the pump beam sizes $W_{p, x}, W_{p, y}$, the absorption coefficient $\alpha_{\text {avg. }}$. In the case of end pumping scheme for axially symmetric distribution of $G_{p}$ and $S_{l}$ the integrals (4), (5) can be solved analytically leading to the well known Laporta's model [2]. However, in our case only numerical results are available. We have limited the scope of analysis only to a pump unit consisting of $1 \mathrm{~cm}$ diode bar with attached cylindrical collimating lens and optional focusing cylindrical lens. We have assumed that $\alpha_{\text {avg }}=0.4 \mathrm{~mm}^{-1}$. The pump beam sizes collimated in $Y$-plane were determined experimentally (see the Table).

T a b l e. Parameters of pumping beam.

\begin{tabular}{lllll}
\hline Direction & $W_{p}[\mathrm{~mm}]$ & $Z_{R}[\mathrm{~mm}]$ & $\Theta_{p}[\mathrm{mrad}]$ & $M^{2}$ \\
\hline$Y$-vertical & 0.3 & 120 & 2.5 & 2.3 \\
$X$-horizontal & 5 & 62.5 & 100 & $\sim 2000$ \\
\hline
\end{tabular}

We intend to estimate the threshold, mode matching efficiency and resulting optical efficiency as a function of cavity and pump volume parameters for three types of pumping scheme given in Fig. 1. The main task of calculations is the choice of proper laser mode geometry and parameters of pump beam with respect to maximization of output power. Let us notice that due to the low incidence angle with respect to the slab 


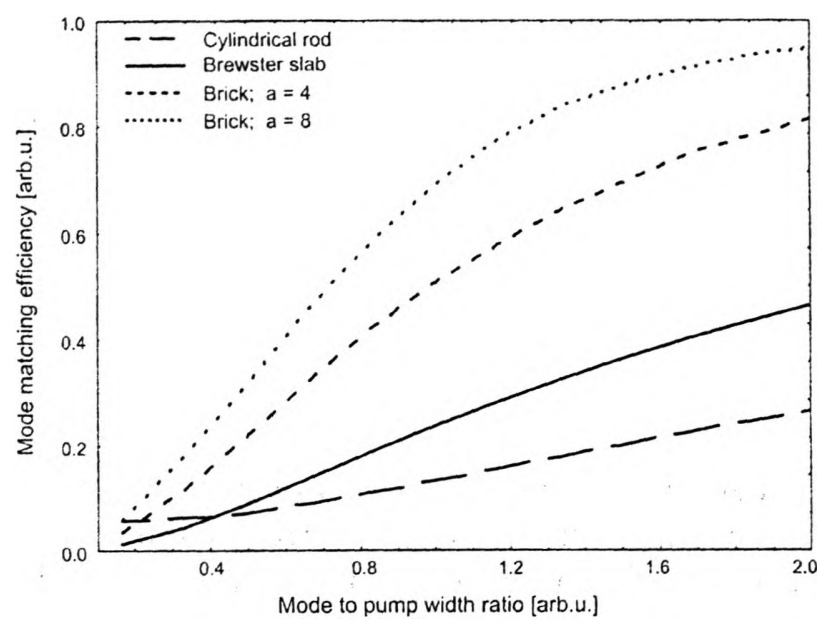

Fig. 2. Mode matching efficiency $v s . W_{0} / W_{p}$; the first three curves correspond to experiments.

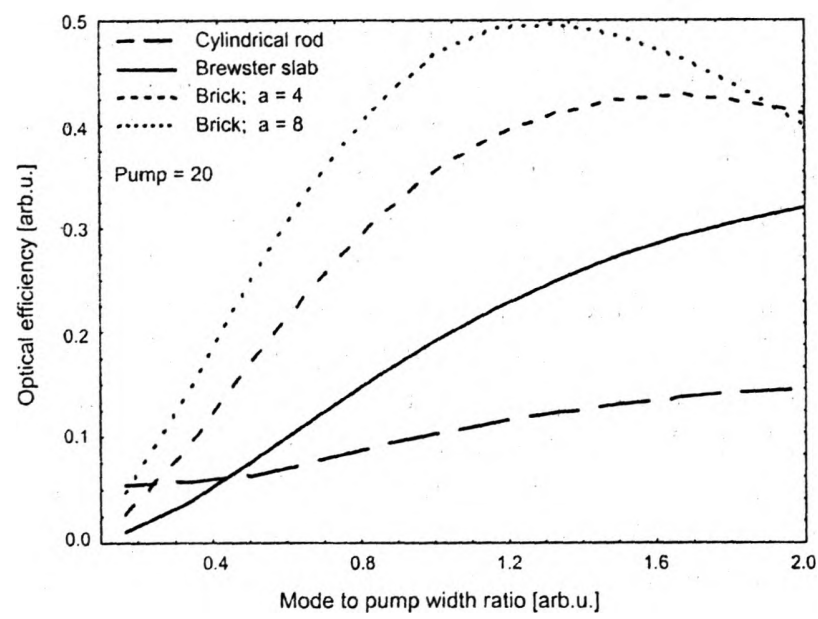

Fig. 3. Optical efficiency $v$. $W_{0}^{\prime} / W_{p}$; the first three curves correspond to experiments.

or brick basis (see Figs. 1b, c), laser mode beam has asymmetrical shape inside the pump volume with high aspect ratio (AR) resulting in enhanced overlapping of pump and laser mode beams for schemes $\mathbf{b}$ and $\mathbf{c}$. The Brewster cut slab (in a case of Brewster slab AR 3) can be considered to some extent as a particular case of a brick type with incidence angle at slab basis corresponding to Brewster cut facets. As shown in Figs. 2 and 3, the threshold as well as mode matching and optical efficiency increase with waist radius $W_{0}$, thus for a given pump power we should find the compromise between these two contrary effects.

The results of numerical analysis were quantitatively confirmed in experiments carried out under $\mathrm{cw}$ pumping (Sec. 5). The best performance in theoretical analysis 
was obtained for type $\mathbf{c}$ (see Fig. 1) with incidence angles of $7^{\circ}$ (parameter $a=8$ ). The brick with incidence angle of $14^{\circ}$ (parameter $a=4$ ) gives a slightly lower performance compared to the previous one, however in experiments we obtained the highest output power for such a case.

\section{Investigations of temperature profiles}

It was found in preliminary characterization that performance of side pumped heads (see Fig. 4) is delimited by thermally induced losses in the gain medium. The beam

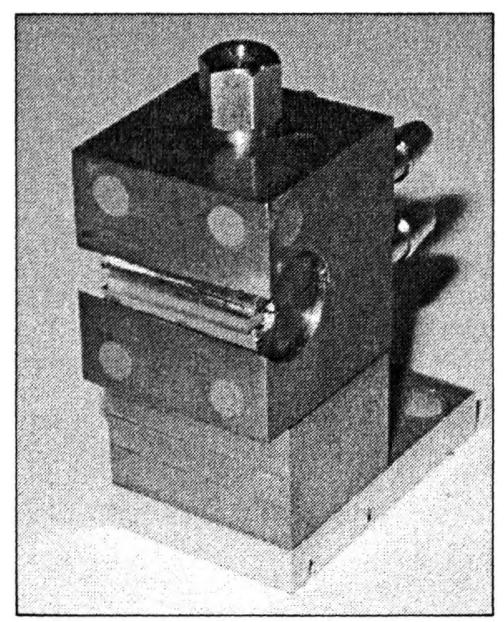

Fig. 4. Photo of the side pumped head.

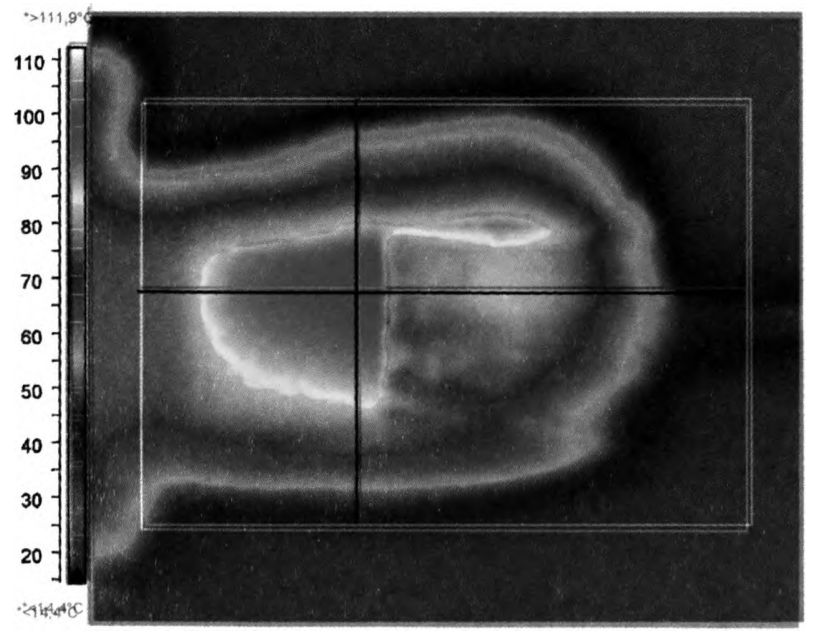

Fig. 5. 2-D temperature profile in the slab at a pump power of $34 \mathrm{~W}$. 


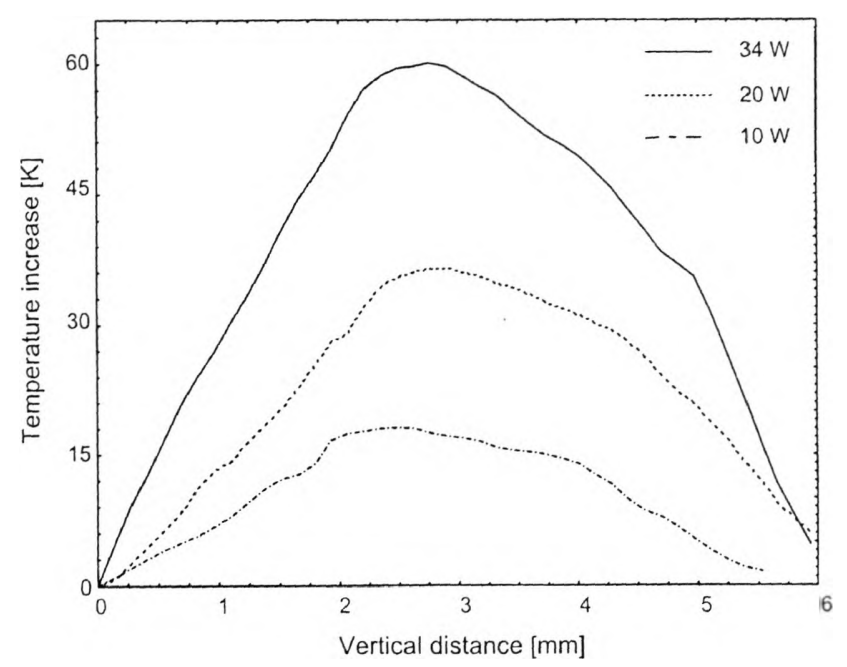

Fig. 6. Temperature increase $v$ s. vertical distance in the middle of the slab at different pump powers.

quality significantly decreased with an increase in average pump power. Thus, we intended to investigate the thermally induced changes of active elements without and with laser generation.

We have tried to examine the temperature profiles across output facets of crystals by means of thermal camera TermaKam SC1000 operating in the $3-5 \mu \mathrm{m}$ wavelength window. Due to big uncertainty in emissivity of dielectrically coated facets the results of temperature measurements are rather quantitative than qualitative (see Figs. 5, 6). We have found that in spite of a significant difference in the shape of rod, slab and brick, the basic optical changes of crystals are determined by asymmetrical shape of absorbed pump density. As mentioned in the previous section, we have used additional cylindrical lens to focus the pump inside the gain element in vertical plane. The pump vertical width was changed in the range of $0.1-0.5 \mathrm{~mm}$, whereas in horizontal plane the width was about $12 \mathrm{~mm}$. Thus the stronger temperature gradients $(>10 \mathrm{~K} / \mathrm{mm})$ and resulting thermal lensing, stresses, thermally induced birefringence occur in the vertical direction. Moreover, due to the differences in crystal geometry and heat contacts the different temperature rise and resulting deformation were observed for each element.

\section{Measurements of depolarization losses}

The main effect of asymmetrical pumping consists in thermally induced anisotropy of gain element. Let us notice that after mounting without pumping the residual anisotropy of crystals was negligible (extinction ratio $<10^{-3}$ ). We have measured the depolarization effects in a scheme as shown in Fig. 7. A linearly polarized probe beam of He-Ne laser, after passing through active medium exactly along the same path as laser mode, goes through the Glan polarizer rotated at $90^{\circ}$ and is observed in far field 


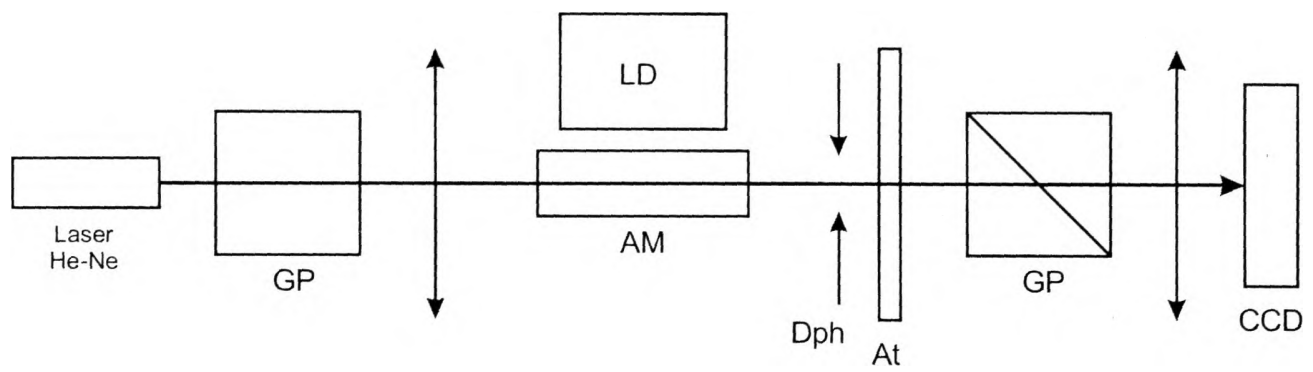

Fig. 7. Experimental set up for investigation of thermally induced depolarization effects: LD - pumping unit with beam forming optics, AM - laser head with active element, Dph - diaphragm, At - variable attenuator, GP - Glan-Taylor polarizer, CCD - camera CCD WAT 902B.

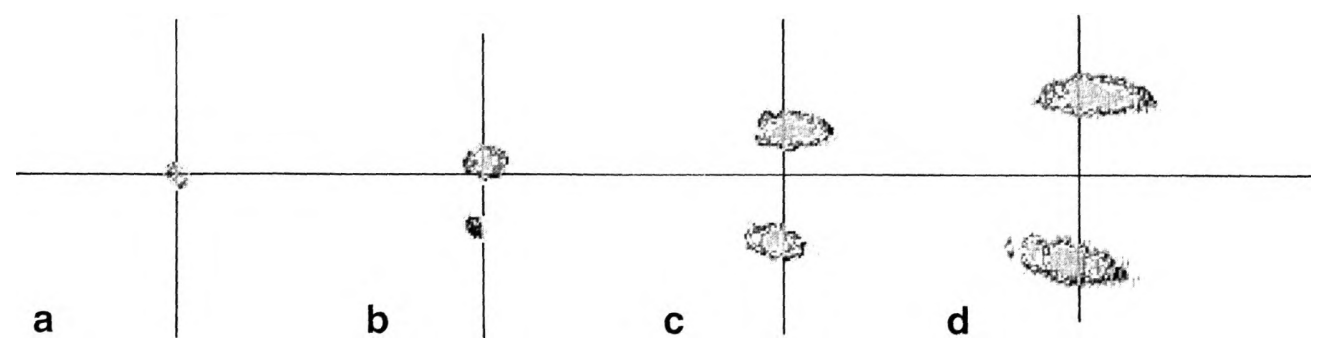

Fig. 8 . The $90^{\circ}$ rotated polarized beam in far field after passing through the brick for several pump powers: $0 \mathrm{~W}(\mathbf{a}), 15.5 \mathrm{~W}(\mathbf{b}), 25.5 \mathrm{~W}$ (c), $34 \mathrm{~W}(\mathbf{d})$.

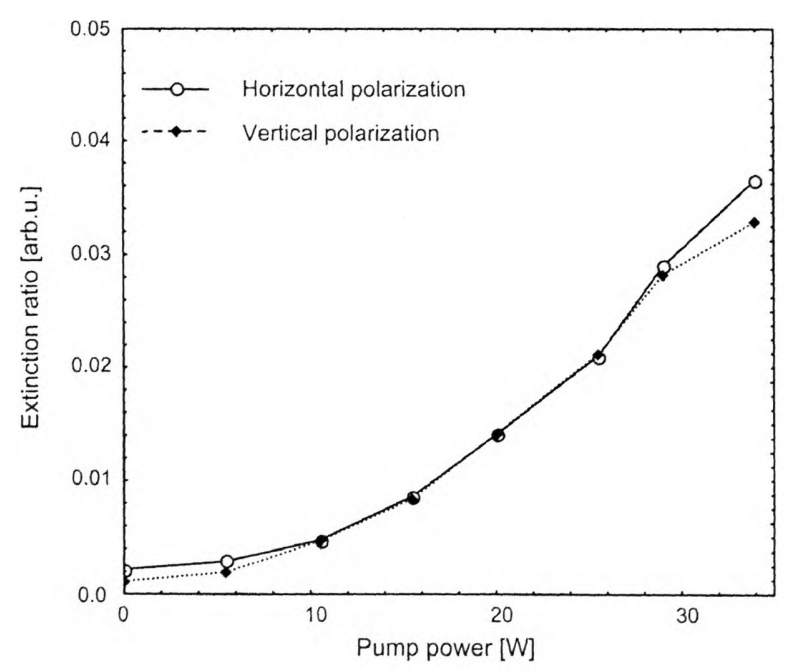

Fig. 9. Extinction ratio vs. pump power for head type a (see Fig. 1).

by means of CCD camera or its power is measured by means of power meter Newport 1525. A very similar effect of splitting of the incident wave into two output beams (see Fig. 8) with orthogonal polarization and separation dependent on average 


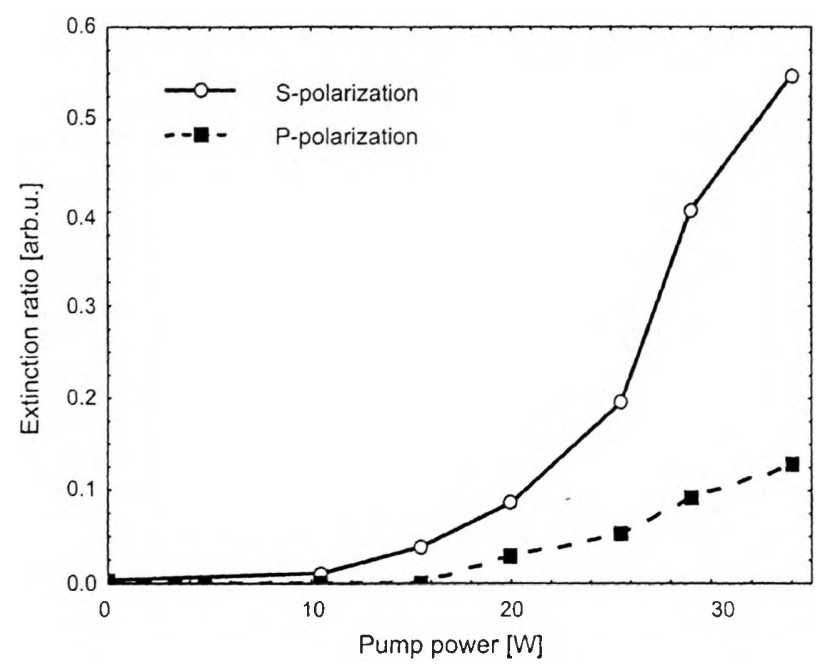

Fig. 10. Extinction ratio vs. pump power for head type c (see Fig. 1).

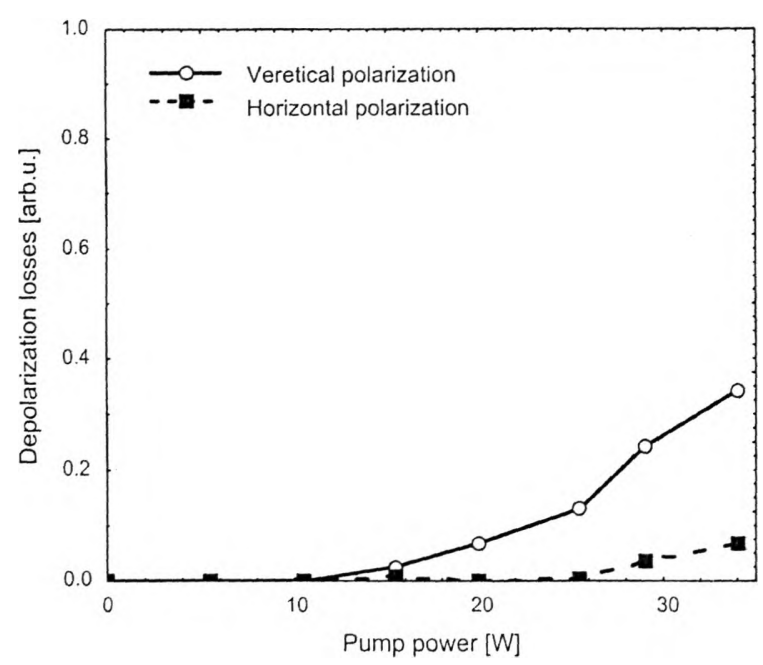

Fig. 11. Depolarization losses vs. pump power for head type a (see Fig. 1).

pump power was observed for each type of laser head. Further, the extinction ratio was measured for different incident polarization states for head types a, c (see Fig. 1). As shown in Figs. 9, 10 the extinction ratio in the case of brick (head type c) is more than 10 times higher compared to cylindrical rod. Thus, the level of depolarization losses is much higher in this case. We have used here the depolarization losses defined as follows:

$$
\delta\left(I_{p}\right)=1-T^{2}\left(I_{p}\right)
$$




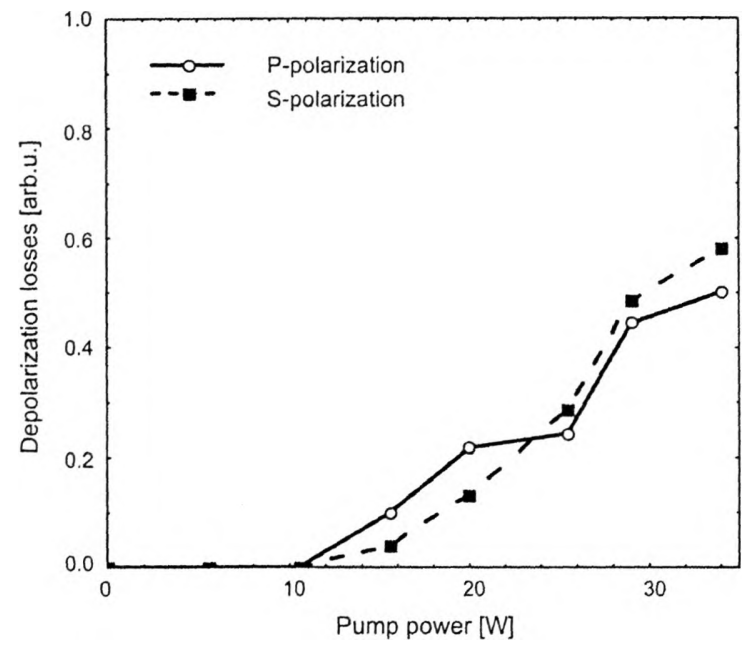

Fig. 12. Depolarization losses vs. pump power for head type c (see Fig. 1).

where $T\left(l_{p}\right)$ denotes the normalized transmission of the system including gain element with parallel oriented polarizers, $I_{p}$ is the pump power. The results of measure-ments of depolarization losses are shown in Figs. 11, 12.

\section{Measurements of output characteristics}

The energetic characteristics were measured for heads mounted in a short cavity of $70 \mathrm{~mm}$ in length (see Fig. 13). Because of the much wider aperture of Brewster slab compared to brick the output beam in this case was highly multimode. We obtained here up to $8 \mathrm{~W}$ of output power (see Fig. 14). In the case of brick (head

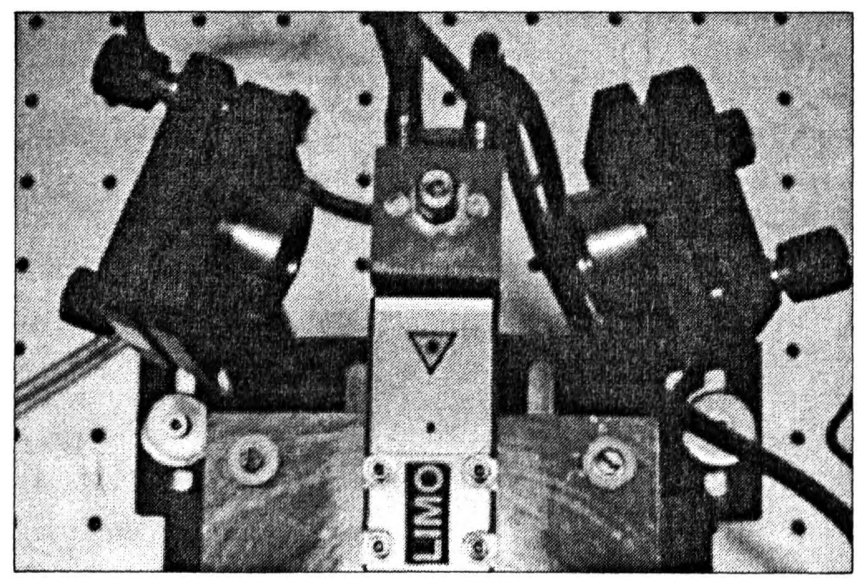

Fig. 13. Photo of cavity with grazing incidence Nd:YAG brick. 


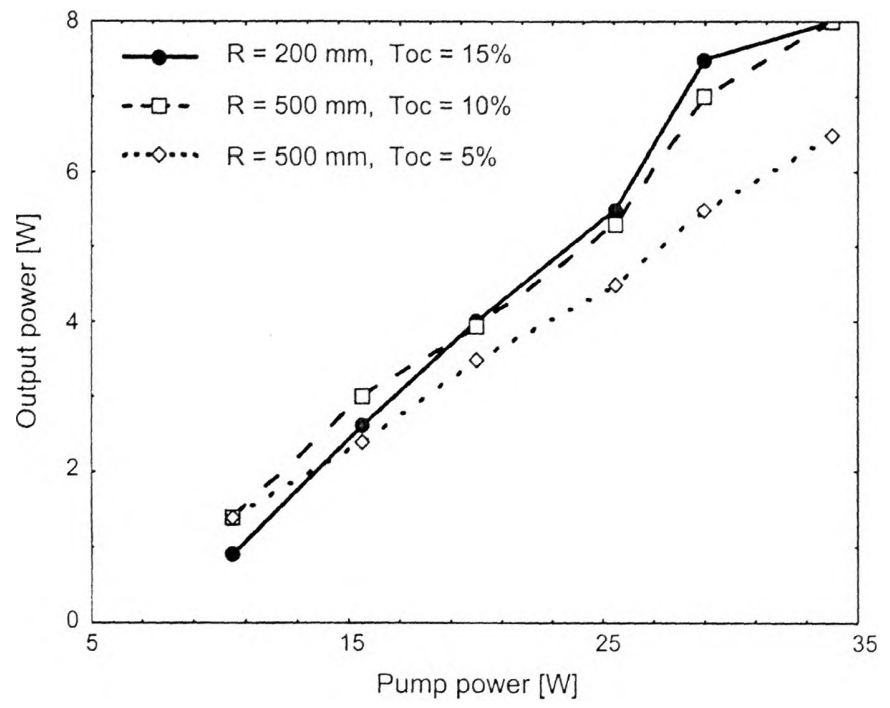

Fig. 14. Output power vs. pump power for Nd:YAG Brewster slab laser.

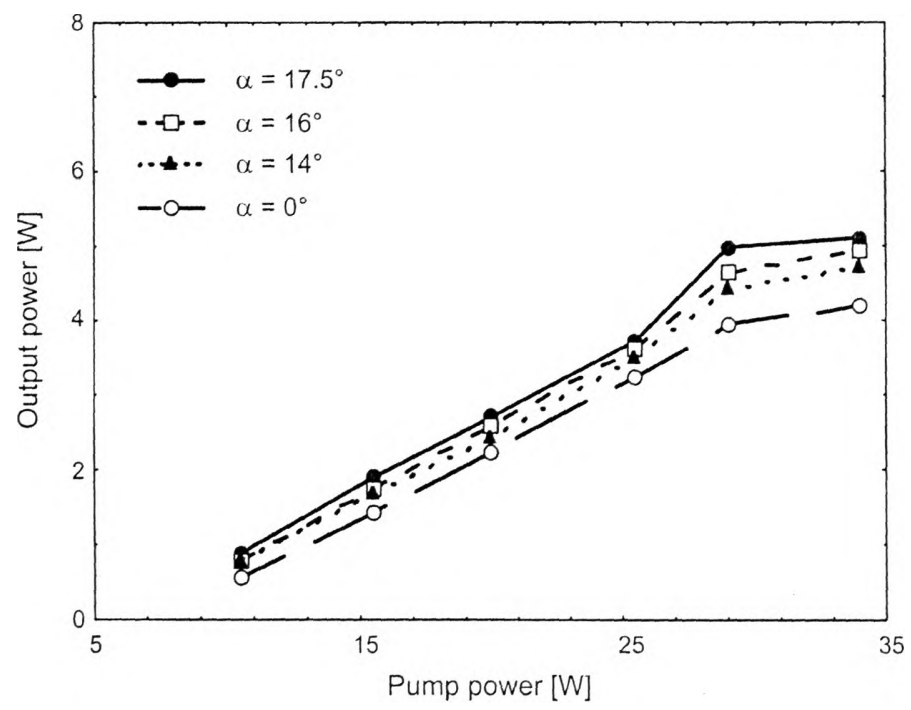

Fig. 15. Output power vs. pump power for Nd:YAG brick laser.

type $\mathbf{c}$ - see Fig. 1) the optimal angle of incidence was first determined (see Fig. 15). The divergence angle of output beam was lower compared to a beam emitted by laser with head type $\mathbf{b}$. The beam quality parameter was estimated to be $M^{2} \cong 2 \times 10$. The lowest output power of $4 \mathrm{~W}$ was obtained for head type a as a result of lower absorption efficiency of cylindrical rod. 


\section{Conclusions}

Theoretical results of the modeling of side pumped heads were quantitatively verified in experiments. The main source of losses in such heads are thermally induced depolarization and astigmatic thermal lensing with complicated higher order aberrations. Thus, such schemes, showing a satisfactory performance for pumping with low duty factor [8]-[10] present much lower quality in the case of $\mathrm{cw}$ pumping with average powers exceeding $20 \mathrm{~W}$ per $1 \mathrm{~cm}$. Application of anisotropic gain media as $\mathrm{Nd}: \mathrm{YVO}_{4}$ or Nd:YAP should give much better results. The worst performance of the head with cylindrical rod is caused by a very narrow pump beam and lower absorption efficiency. With uniform pump density distribution such a scheme should give the best performance, however it requires application of few (3-5) pumping bars being beyond the scope of the present work. Asymmetry in heating and cooling of slab and brick elements is the main factor delimiting the direct application of such elements in $\mathrm{cw}$ high power lasers. The best choice in our opinion for a single pumping diode bar is the head of type c, see Fig. 1) with an active medium of brick shape. The proper heat contacts, good matching of the pump wavelength to absorption peak of gain medium and careful design and technology of brick fabrication are the major factors leading to improvement of such a type of laser head.

Acknowledgments - This work was partly supported by Polish State Committee for Scientific Research (KBN) under the projects 0T00A06519 and 4T11B02724. We would like to thank Drs R. Dulski, M. D'browski for help in thermal investigations.

\section{References}

[1] Fan T.Y., Byer R.L., IEEE J. Quantum. Electron. 24 (1988), 895.

[2] Laporta P., Brussard M., IEEE J. Quantum. Electron. 27 (1991), 2319.

[3] Tidwell S.C., Seaman J.F., Bowers M.S., Cousins A.K., IEEE J. Quantum. Electron. 28 (1992), 997.

[4] Clarkson W.A., Hanna D.C., Opt. Lett. 21 (1996), 375.

[5] Marshall L.R., Kaz A., Burnham R.L., Opt. Lett. 17 (1992), 186.

[6] Baer T. M., Head D.F., Gooding P., Kintz G.J., Hutchinson S., IEEE J. Quantum. Electron. 28 (1992), 1131.

[7] Brauch U., Schubert M., Opt. Commun. 117 (1995), 116.

[8] Kirchmayer W., Herrmann H., Nagel E., Haring R., Streicher J., Werner Ch., Mehnert A., Halldorsson Th., Heinmann S., Peuser P., Schmitt N.P., Opt. Laser Technol. 25 (1993), 283.

[9] JABCZYŃSKI J.K., ŻENDZIAN W., Opt. Appl. 27 (1997), 205.

[10] Bernard J.E., Alcock A.J., Opt. Lett. 18 (1993), 968. 